\title{
Pasireotide shows promise for the treatment of acromegaly
}

Pasireotide is a promising novel treatment for acromegaly, according to the findings of a randomized phase II trial.

Pasireotide is a somatostatin analog with high affinity for both somatostatin receptor subtypes (types 2 and 5) expressed by most growth-hormonesecreting pituitary tumors. Consequently, pasireotide has the potential to be a more effective therapy for acromegaly than the somatostatin analogs octreotide or lanreotide that have low affinity for the type 5 receptor.

Sixty patients with active acromegaly received octreotide $100 \mu \mathrm{g}$ three times daily for 28 days and then pasireotide at $200 \mu \mathrm{g}, 400 \mu \mathrm{g}$ and $600 \mu \mathrm{g}$ twice daily in random order, each for 28 days. The researchers defined a biochemical response to treatment as a growth hormone level of $\leq 2.5 \mu \mathrm{g} / \mathrm{l}$ and a level of insulin-like growth hormone 1 normalized to that of controls.

A biochemical response was achieved in $9 \%$ of patients after 28 days of octreotide, a low response rate that may partly be due to the possible inclusion of patients who were resistant to previous treatment with somatostatin analogs. After 3 months of treatment with pasireotide 200-600 $\mu$ g, about one-third of patients achieved a biochemical response and tumor volume decreased by $>20 \%$ in $39 \%$ of patients. Pasireotide was generally well tolerated.

"The potential of pasireotide will only become clear once results are available from the ongoing randomized, phase III study comparing the long-acting release formulations of octreotide and pasireotide," concludes lead researcher Stephan Petersenn of the University of Duisburg-Essen, Germany.

\section{Carol Wilson}

Original article Petersenn, S. et al. Pasireotide (SOM230) demonstrates efficacy and safety in patients with acromegaly: a randomized, multicenter, phase II trial. J. Clin. Endocrinol. Metab. 95, 2781-2789 (2010) 